\title{
Awareness on COVID -19 outbreak and its preventive strategies among a group of Sri Lankan social media users: A Descriptive Cross-Sectional Study Perera KYD ${ }^{1}$, Karunarathne $\mathrm{HMAH}^{2}$, Dileka WSK ${ }^{2}$, Dissanayake $\mathrm{Y}^{3}$, Amadoru $^{1}$
}

\begin{abstract}
Introduction: Corona Virus Disease-19 (COVID-19)discovered in December-2019 soon became a pandemic and was declared a global public health emergency.

Objectives: The study assessed the public awareness on COVID-19 outbreak among a group of Sri Lankan social media users.

Methods: This cross-sectional survey was conducted in late March-2020,using a self-administered online-questionnaire shared in social media.

Results : Out of 174 respondents, 97 (55.7\%) were male and the mean age was 36.1 years. Most were from western province $(n=109,62.7 \%)$ and with higher education (64.5\%). All ofthe common transmitting modes were identified by only 141 (81\%). Respondents identified fever (97.7\%) and Cough-93.7\% as features of COVID while $131,(75.3 \%)$ knew about asymptomatic patients. Even though170 (97.7\%) knew the duration of quarantine period $24.6 \%$ had a misconception on indications for quarantine. Majority $(158,90.8 \%)$ thought the herbal drinks are beneficial. Majority $(n=153,87.9 \%)$ knew the indications for using masksand $165(94.8 \%)$ were aware about social distancing. Majority $(n=162,93.1 \%)$ were satisfied about the initial COVID-19 control-measures by the government.

Discussion: The level of overall knowledge on the COVID-19 was satisfactory among the participants with some deficiencies including all the modes of transmission. This shows the space for further awareness for controlling the virus better.

Key words: COVID-19, Modes of transmission, Public awareness, Social media use
\end{abstract}

\section{Introduction}

According toWorld Health Organization(WHO), emerging infectious disease is defined as newly identified and previously unknown infections which cause public health problems either locally or internationally. These include HIV infections, Severe Acute Respiratory Syndrome (SARS), Lyme disease, hantavirus, dengue fever, West Nile virus and the Zika Virus etc. $^{1}$

Corona Virus Disease- 19 (COVID -19) is a newly discovered emerging infectious disease caused by the most recently discovered coronavirus, SARS CoV2.

1. Registrar in Medical Administration, Ministry of Health, Sri Lanka

2. Acting Consultant Venereologist

3. Registrar in Medical Microbiology

Corresponding Author:

K Y Dhanushka Perera

Email:dhanushkaperera@ymail.com

(iD) https://orcid.org/0000-0002-7978-3284
This new virus and disease were unknown before the outbreak reported as cluster of cases of pneumonia in Wuhan, China, in December 2019.Inlate January with the spread of the disease, the WHO Director- General declared the novel coronavirus outbreak (2019-nCoV) a Public Health Emergency of International Concern (PHEIC). ${ }^{2}$

The disease spreads primarily from person to person through small droplets from the nose or mouth, expelled when a person with COVID- 19 coughs, sneezes or speaks. These droplets are relatively heavy, do not travel far and quickly sink to the ground. People can become infected- by touching the objects or surfaces where these droplets land, then touching their eyes, nose or mouth. ${ }^{3}$

Once infected, the most common symptoms of COVID -19 are fever, dry cough and tiredness. Other symptoms that are less common and may affect some patients include aches and pains, nasal congestion, headache, conjunctivitis, sore throat, diarrhea, loss of taste or smell. These symptoms are usually mild and begin gradually. Some people become infected but only have very mild symptoms. 
Most people (about $80 \%$ ) recover from the disease without needing hospital treatment. Around 1 out of every 5 people who gets COVID- 19 becomes seriously ill and develops difficulty breathing. Older people and those with underlying medical problems like high blood pressure, heart and lung problems, diabetes or cancer, are at higher risk of developing serious illness. ${ }^{4-6}$

Practicing 'hand and respiratory hygiene' and maintaining the social distance at least a 1-meter distance between two persons are the best ways to protect from the disease. Hence, primordial prevention with health education to promote safe and hygienic health practices in day to day life activities is the most suitable mode of prevention considering the socio-economic impact the disease can cause. ${ }^{7}$

By end October 2020, most (216) countries, areas or territories are affected with the COVID -19, with 46 million confirmed cases and just under 1.2 million confirmed deaths globally with adisease specific fatality rate is $3.16 \%{ }^{8}$

After 1streported local case of COVID -19during early March 2020,multidisciplinary approach of COVID- 19 prevention task force. Those activities raised the awareness about COVID -19 among the Sri Lankanpopulation. Health educational and promotional activities were taken place early. Despite these measures, By the beginning of November 2020, after nine months of emergence of the infection in the country, Sri Lanka had 11,774 total confirmed cases, with 23 deaths giving rise to a disease specific fatality rate of $0.20 \%{ }^{9}$ Community transmission of the disease was not declared by the health authorities because the new cases could be linked to previous patients.

Few studies exploring the awareness on COVID-19 infection among general public emerged by mid-2020. Many of the studies dem-

onstrated a satisfactory awareness. ${ }^{10-12}$

As far as we know no study has been published on the topic from Sri Lanka.

\section{Objective}

The objective of this study wasto assess the public awareness on COVID -19 outbreak and its preventive strategies among the Sri Lankan population.

\section{Materials and Methods}

This cross-sectional survey was conducted from March25th to April 1st, the week immediately after the initiation oflockdownsinSri Lanka. Because it was not feasible to do a community-based national sampling survey during this special period, the data collection was carried out using an online questionnaire. Relying on the authors' networks with people living in the country, one-page recruitment poster was posted/reposted on groups of their WhatsApp,Viberand Facebook accounts. This poster contained a brief introduction on the background, objective, procedures, voluntary nature of participation, declara-

Table 1- Socio demographics of the sample

\begin{tabular}{|lll}
\hline Gender & Number & \% \\
Male & & \\
Female & 97 & $55.7 \%$ \\
& 76 & $43.7 \%$ \\
Age group & 173 & $100 \%$ \\
<20 years & & \\
20-29 years & 2 & $1.1 \%$ \\
30-39 years & 23 & $13.2 \%$ \\
$40-49$ years & 110 & $63.2 \%$ \\
50 -59 years & 29 & $16.7 \%$ \\
60 years and above & 6 & $3.4 \%$ \\
& 4 & $2.3 \%$ \\
District of Residence & & \\
Colombo & & \\
Gampaha & 76 & $43.7 \%$ \\
Matara & 33 & $19.0 \%$ \\
No Response & 21 & $12.1 \%$ \\
Anuradhapura & 12 & $6.9 \%$ \\
Kalutara & 5 & $2.9 \%$ \\
Other & 5 & $2.9 \%$ \\
& 22 & $12.5 \%$
\end{tabular}

Educational Qualifications

$\begin{array}{lll}\text { Grade } 11 \text { or less } & 1 & 0.6 \% \\ \text { GCE O / L } & 8 & 4.6 \% \\ \text { GCE Advanced Level } & 20 & 11.5 \% \\ \begin{array}{l}\text { Diploma or other } \\ \text { professional qualification }\end{array} & 31 & 17.8 \% \\ \begin{array}{l}\text { University degree } \\ \text { Postgraduate or higher }\end{array} & 57 & 32.8 \% \\ \text { Total } & 52 & 29.9 \% \\ & 174 & 100.0 \%\end{array}$

tions of anonymity and confidentiality, and notes for filling in the questionnaire, as well as the link and quick response (QR) code of the online questionnaire. Persons who were of Sri Lankan nationality, aged 18 years or more, understood the content of the poster, and agreed to participate in the study were instructed to complete the questionnaire via clicking the link or scanning the QR code.

Participants had to answer a yes-no question to confirm their willingness to participate voluntarily. After confirmation of the question, the participant was directed to complete the self-administered questionnaire.

\section{Results}

Socio-demographic characteristics-

One hundred and 
seventy four (174)participants responded to the self-administered questionnaire. Mean Age of the respondents was 36.1 years (range-10-69). Majority were Male- ( $n=97,55.7 \%$ ) withone respondent not disclosing the gender. Majority of participants were from Colombo ( $n=76,46.9 \%$ ), followed by Gampaha $(n=33,20.4 \%)$ and Matara( $n=21,13 \%)$. Out of the respondents, 57 (33.7\%) were degree holders while 52 (30.8\%) had Postgraduate diploma or other higher education qualifications( $n=31,18.3 \%)$. Twenty $(11.8 \%)$ participants were educated up to GCE Advanced Level. Socio demographic details are shown in table 1.

Table 1- Socio-demographic Characteristics of the participants

\section{Knowledge on COVID-19}

The respondents' knowledge on COVID 19 infection and related factors is shown in table 2 . All 174 participants correctly identified Corona as a Virus and that the current situation is being called a pandemic. However, 163 (94.8\%) knew that a global public health emergency had been declared.

The most common Transmitting modes were identified by only 141 (81\%). Some $\quad(n=32,19 \%)$ didn't think that close proximity and common use of personal belongings can cause virus to spread.Others $(n=31)$ thought the virus can only transmit through respiratory Ta

1

Incubation period

2-14 days

2-4 weeks

1-2 days

Quarantine period

14 days

21 days

7 days

Symptoms of COVID*

Dry Cough

Nasal Congestions

Throat pain

Diarrhea

Fever

Breathing difficulties

Yes

Don't know

At risk communities*

diseases ie Diabetes

'Children

Youth population

Wearing face masks

Food safety

Avoiding large crowds

People who stay at home

Close contacts of patients
Table 2- Knowledge about the Co

Can COVID patients be asymptomatic?

droplets by air.

The majority of the respondents were able to identify the symptoms of the disease except for diarrhoea. Eighty-six (49.4\%) participants thought diarrhoea can be a symptom. The participants identified breathing difficulties as the commonest symptom (98.9\%) followed by fever (97.7\%), throat pain $(96 \%)$, dry cough $(93.7 \%)$ and nasal

Family members of patients $\quad 162 \quad 93.6 \%$

Health care workers $\quad 134 \quad 77.5 \%$

No change $\quad 158 \quad 91.3 \%$

General public $\quad 3 \quad 1.7 \%$

Elderly population $\quad 171 \quad 98.3 \%$

$\begin{array}{lll}\text { Persons with chronic } & 162 & 93.1 \%\end{array}$

$73 \quad 42.0 \%$

$8.0 \%$

Table 3-Knowledge on preventive aspects of COVID 19

Number \%

$172 \quad 99.0 \%$

Avoid touching face frequently 169

Frequent handwashing with soap and water $171 \quad 98.8 \%$

Cleaning frequently touched surfaces $\quad 145 \quad 83.8 \%$

$41.6 \%$

$\begin{array}{ll}72 & 41.6 \% \\ 172 & 99.4 \%\end{array}$

When should a person wear a facemask*

Taking care of COVID patients

$16696 \%$

When someone shows symptoms of COVID

171

14

$98.8 \%$

$8.1 \%$

Who are indicated for quarantine*

Family members of patients

164

$94.2 \%$

Returnees from a COVID prevalent country

People living near quarantine center congestions $(58.6 \%)$

* Multiple responses were possible from one participant

Incubation period was correctly identified by 155 (89.1\%) as 2 to 14 days. Seventeen (9.8\%) thought that it is two weeks to four weeks.

Almost all $(n=173)$ thought that travelling history is important in managing COVID patients.

Majority $\quad(n=131$, $75.3 \%$ ) knew that COVID infected patients can be asymptomatic. However, it is noteworthy that around a quarter of the participants did not know this important fact.

\section{Quarantine process}

When it comes to knowledge on the quarantine period, the majority $(n=170$, $97.7 \%) \quad$ correctly identified it as 14 days and three respondents thought its 21 days while one thought its 7 days.

The vast majority of the participants correctly identified that the family members 
( $n=168,96.5 \%)$ and close contacts of COVID patients ( $n=164,94.2)$ should be quarantined. Despite repeated media educating the public, 26 (15.2\%) thought persons coming from a COVID19 prevalent country need not be quarantined.Forty-five (24.6\%) thought that residents around the infectious disease hospital treating COVID19 patients should be quarantined.

\section{Treatment for COVID 19}

The majority ( $n=144$, $82.8 \%$ ) knew that there are no particular drugs for COVID and 20 (11.5\%) didn't know about drugs and 10 thought there is a particular drug.

Majority ( $n=158,90.8 \%)$ thought the herbal drinks are beneficial for recovery from COVID 19 and only 14 thought that those will make no difference. How-

Table 4- Factors that may affect prevention and the prognosis of COVID 19

\begin{tabular}{|cll|}
\hline & Number & $\%$ \\
Alcohol & \multicolumn{1}{l|}{} \\
Beneficial & 5 & $2.9 \%$ \\
Harmful & 117 & $67.2 \%$ \\
No change & 9 & $5.2 \%$ \\
Smoking & & \\
Beneficial & 1 & $0.6 \%$ \\
Harmful & 173 & $99.4 \%$ \\
No Change & 0 & \\
Plenty of Water & & \\
Beneficial & 168 & $96.6 \%$ \\
Harmful & 3 & $1.7 \%$ \\
No Change & 0 & \\
Herbal Drinks & & \\
eg- Corianrum sativum/Kottamalli & & \\
Beneficial & 158 & $90.8 \%$ \\
Harmful & 1 & $0.6 \%$ \\
No change & 14 & $8.0 \%$ \\
\hline
\end{tabular}

ment to control COVID, 162 (93.1\%) were satisfied about the level of commitment from the government with most $(n=86)$ among them feeling very satisfied. Nine (5.2\%) thought the response from the government was average and 3 believed that the measures taken by government is unsatisfactory.

\section{Discussion}

Social media plays a big role in disseminating public health related information throughout the communities worldwide. It was evident that online sources played a major role in informing the general public in the prevailing COVID 19 epidemic as well. However, there was no understanding of the level of awareness on COVID 19 epidemic related information among the social media users. ever, smoking was identified by $173(99 \%)$ as harmful.

\section{Preventive measures for COVID}

Majority ( $n=153,87.9 \%$ ) correctly identified the indications for using masks as advised by the health authorities at the time of data collection. Fifteen (18.9\%) thought it is best to use masks at all times irrespective of other conditions.

When it comes to the minimal distance that should be maintained between two individuals to prevent transmission of COVID, 165 (94.8\%) identified the minimal distance as one meter while nine thought it is two meters.

Majority ( $n=154,88.5 \%)$ ) selected 20 seconds as recommended duration that should be spent for hand-washing while some thought the minimal time should be 2 minutes $(n=11)$ and 20 minus $(n=8)$ respectively.

On inquiry about factors that may have a beneficial or harmful effect on prevention / development of the disease, 117 (67.2\%) identified alcohol as harmful while $5(2.9 \%)$ thought its beneficial and 9 thought there is no difference. Herbal drinks were thought to be beneficial by the majority with $90.8 \%$ selecting it as beneficial.

Perceptions on governments response on managing COVID pandemic in Sri Lanka With regards to the initial measures taken by the govern-
This study revealed thatlevel of knowledge on the COVID 19 was found to be relatively high among the social media users who responded to the questionnaire.

All the participants were aware of the pandemic and the fact COVID is caused by a virus. The majority were able to identify the incubation period and the transmission dynamics of the virus. However, only $81 \%$ of the participants were able to identify all the modes of transmission. Inability to identify all the modes of transmissions was a common deficiency of the general public as found in international studies as well. ${ }^{10-12}$ This shows that further awareness should be carried out on the modes of transmission of the disease. This is vital in battling the pandemic since the knowledge on transmission dynamics plays a major role in preventing spread of the disease Satisfactory level of awareness was also demonstrated on treatments, preventive measures and quarantine process of COVID 19. This may be due to the fact that there were lengthy discussions and debates going on regarding treatment options and the quarantine process.

There was a strong debate going ononline platforms regarding herbal drinks that may play a major role in preventing as well as giving a good prognosis for the patients with COVID 19. The patients were encouraged to drink herbal drinks like Kottamalli/ Coriander(Coriandrum sativum)by various institutions. 
It seems that these inputs have had an impacton the participants, which is seen by $90.8 \%$ of the participants believing that herbal drinks are beneficial. The fact that alcohol is used to deactivate the virus may have been the reason for 5 participants to comment that alcohol may be beneficial. This is a clear indicator that the online sources and mainly the social media can influence the thinking process of its users.

It was obvious that the participants thought that the initial government response towards the COVID 19 was very satisfactory. Only a few had thoughtotherwise. This may be explained by the fact that the number of COVID 19 cases in the county was relatively low with only 3000 cases being identified within the first 6 months. ${ }^{9}$

Since this study was carried out in the early days of the pandemic, the general public would not have received inputs on the disease as at the time of writing in November 2020.One of the major limitations in this study was that the participants were restricted to persons with online access. The same fact must have resulted in selection of a sample with a higher than average level of education and access to public health information.

Even though this study demonstrated satisfactory level of awareness on the COVID 19, the results indicate that there is room for improvement. Hence, it is recommended to strengthen the public awareness programmes. Further, it is recommended to carry out more representative studiesamong general public to understand the status better.

\section{References}

1. World Health Organization, Regional Office for SouthEast Asia. ((2014)|. A brief guide to emerging infectious diseases and zoonoses. WHO Regional Office for South-East Asia. https://apps.who.int/iris/handle/10665/204722

2. Ji W, Wang W, Zhao X, Zai J, Li X. Cross-species transmission of thenewly identified coronavirus 2019nCoV.J MedVirol.(2020) 92:433-40

3. Cai J, Sun W, Huang J, Gamber M, Wu J, He G. Indirect virus transmission in cluster of COVID-19 cases, Wenzhou, China, 2020. Emerg Infect Dis. (2020) 26:10.3201/eid2606.200412.

4. Huang C, Wang Y, Li X, Ren L, Zhao J, Hu Y, et al. . Clinical features of patients infected with 2019 novel coronavirus in Wuhan, China. Lancet. (2020) 395:497-506. 10.1016/S0140-6736(20)30183-5

5. Azer SA, COVID-19: pathophysiology, diagnosis, complications and investigational therapeutics,
New Microbes New Infections (2020) 10.1016/ j.nmni.2020.100738

6. Gulati A, Pomeranz C, Qamar Z, Thomas S, Frisch D, George G, Summer R, DeSimone J, Sundaram B. A Comprehensive Review of Manifestations of Novel Coronaviruses in the Context of Deadly COVID-19 Global Pandemic. Am J Med Sci. 2020 Jul;360(1):5-34.

7. I.K. Lee, C.C. Wang, M.C. Lin, C.T. Kung, K.C. Lan, C.T. Lee, Effective strategies to prevent coronavirus disease2019 (COVID-19) outbreak in hospital, J. Hosp. Infect. (2020), https://doi.org/10.1016/j.jhin.2020.02.022. Advance online publication

8. WHO coronavirus disease (COVID-19) dashboard. Geneva: World Health Organization, 2020. Available online: https://covid19.who.int/ (last accessed on 01.November.2020]).

9. Health Promotion Beareau, Covid 19 Dashboard. 2020. Available online: https://hpb.health.gov.lk/ covid19-dashboard/(lastaccessed on 04.November.2020]).

10.Parikh AP, Shah BV, Phatak AG, Nimbalkar SM. COVID-19 pandemic: knowledge and perceptions of the public and healthcare professionals. Cureus. (2020) 12:e8144. 10.7759/cureus.8144

11.Al-Rasheedi M, Alhazmi Y, Mateq Ali A, Alrajhi M, Samah Alharbi N, Alsuhaibani S, Mohammed A, Alharbi. GeneralPublic and healthcare providers awareness of Coronavirus (COVID-19) in Qassim Region, Saudi Arabia.Saudi J Biol Sci. 2020 Sep 10. doi: 10.1016/ j.sjbs.2020.08.035.

12.Modi PD, Girija N, Uppe A, Modi J, Tuppekar B, Gharpure AS, Langade D , COVID-19 Awareness Among Healthcare Students and Professionals in Mumbai Metropolitan Region: A QuestionnaireBased Survey Cureus 2020 Apr 2;12(4):e7514. doi: 10.7759/cureus.7514. 